\title{
A Sustainable Human Centered Design Framework Based on Human Factors
}

\author{
H. Onan Demirel ${ }^{1}$ and Vincent G. Duffy ${ }^{1,2,3}$ \\ ${ }^{1}$ School of Industrial Engineering \\ ${ }^{2}$ School of Agriculture and Biological Engineering \\ ${ }^{3}$ Regenstrief Center for Healthcare Engineering \\ Purdue University \\ 47907 West Lafayette, IN, USA \\ \{hdemirel, duffy\} @purdue.edu
}

\begin{abstract}
Since humans are creators and users of artifacts and processes, and are constrained by limited resources, a sustainable approach with human element in the center of the development cycle is vital today. Managing and understanding technical and analytical issues about human element in design process is a core challenge for practitioners to develop and improve design strategies that serves to accommodate human needs, abilities and limitations. Although attention has increased in recent decades, direct progress towards a sustainable product development is still not apparent. Methods for sustainability are mostly applied as post-processing activities to safeguard nature, however a pro-active approach is necessary. A sustainable Human Centered Design platform provides an alternative approach to deal with the degeneration of the ecology while providing alternative criteria for considering customer requirements. This paper focuses on improving product design by considering Human Factors as a medium to integrate Sustainability and Human Centered Design strategies. The emphasis in this framework is to introduce Human Factors aspects of product design early in the concept generation phase, provide a modular platform to allow integration of domain knowledge and technologies and to enhance the product development, and overall well being of human element in design cycle.
\end{abstract}

Keywords: Human Centered Design, Sustainability, Human Factors, Product Design, Digital Human Modeling, Ergonomics.

\section{Introduction}

The design field is regarded as an activity or a process domain rather than a scientific discipline. It is the convergence of scientific, non-technical and social processes. In daily communication, the term design is mostly associated with crafting and/or constructing artifacts, however it is actually a thought process for creating tangible and intangible products, services and processes. Depending on the field of interest there are prominent differences at the extremes [1,2]. Design may encompass vast variety of context, knowledge and experiences from different domains. Design outcomes may range from vehicles, machines, consumer products and artistic items at the one end of 
the spectrum, to software, interactive systems and thought processes at the other end. This broad perspective brings the need of considering both technical and nontechnical aspects, which includes; form and functionality, as well as means of integration with advancements in technology, engineering, artistic trends, sciences, society, policy making, education, sustainability, business and education (Fig.1).

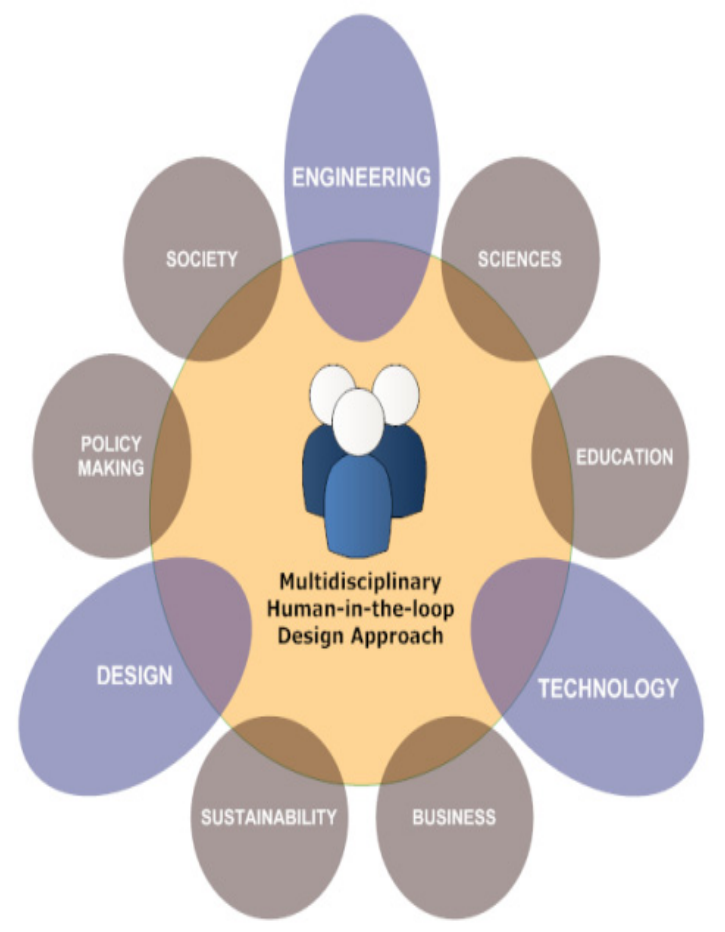

Fig. 1. Multidisciplinary Human-in-the-loop Design Approach

Global marketing competition and rapidly changing customer demands require innovative design, manufacturing and maintenance techniques to provide high level customer appreciation and safety for the end user [3]. The need for optimizing trade-offs among changing consumer attributes (customization, variety of options), convergence of new technologies (nanotechnology, biotechnology), ecological awareness (green design, recycling), financial concerns has challenged designers and motivated design industry to seek alternative methods. There are many methods introduced, however the majority of these methods focus on the specific parts and/or elements of the design process rather than offering a holistic approach. This paper provides a brief introduction to a holistic framework based on Human Factors, which integrates different domains and design elements to form a sketch-toanalysis type design process. 


\section{Human Element and Sustainability in Product Design}

Human element is the core of any design process. Since humans are the creators and users of artifacts and processes, and are constrained by limited resources, a sustainable approach with the human element in the center of the development cycle is vital today. It is challenging to find an optimum in such a diverse, complex and dynamic system, which encompasses human and nature. However understanding common elements and starting to develop a product design process around these elements have potential to build a robust design platform. A sustainable Human Centered Design platform provides an alternative approach to deal with the degeneration of the ecology while providing alternative criteria for considering customer requirements [4,5].

\subsection{Human Centered Design}

Introducing a new product to market comes with high risks. A product with technology innovation may result in market failure, if it does not follow customer requirements [6,7]. Understanding customer attributes and following consumer trends have became major success drivers between products. Designers in quest to find versatile strategies to deliver innovative products in high volumes with short lead times at a reasonably low cost, while satisfying customer wants and meeting environmental rules and regulations. This beneficial, yet hard to achieve goal brought challenges for product design $[6,7,8]$.

Human Centered Design integrates different technical and social fields of expertise to enhance well-being of people by improving product-user interaction, increasing usability, safety and efficiency. Definition of Human Centered Design may differ depending on the context, however the approach involves techniques which communicate, interact and stimulate people involved in product interaction and continuously improve the product characteristics depending on needs, abilities and limitations of users $[9,10]$. Although above definition is very similar to Human Factors, Human Centered Design is not a scientific domain but a design methodology, which captures variability between users and accommodates these differences efficiently in product design, which would satisfy users from different physical and cognitive perspectives. Managing and understanding technical and analytical issues about the human element in the design process is a core challenge for practitioners to develop and improve design strategies that serves to accommodate human needs, abilities and limitations [11].

\subsection{Sustainability}

The definition of sustainability may vary, depending on the context, the domain and practitioner/author's point of view. Topics in media regarding sustainability focus on protection, preservation and conservation of environment and nature. However, in theory and application sustainability has a much larger coverage and impact area in daily living and should not be solely regarded as a post-process activity to safeguard ecology. Sustainability is a pro-active approach for increasing the quality of living, 
which considers wide range of different context and domain knowledge. According to World Commission on Environment and Development sustainability can be defined as "meeting the current needs without compromising ability of future generations to meet their own needs" [12]. Also The Rio Declaration on Environment and Development's first principle is that "human beings are at the center of concerns for sustainable development. They are [entitled] to a healthy and productive life in harmony with nature" [12].

Above definitions clearly show that the core activity and focus of the sustainability is the human element and it requires a pro-active approach, which would improve quality of human life by considering every aspect of life that would have inner, inter and trans-disciplinary relationships with the human element. Today, earth's population consumes natural resources faster than the Earth replenishes them [12]. Methods for sustainability are mostly applied as post-processing activities to safeguard nature, however a pro-active approach is necessary. Although attention has increased in past decades, a direct progress towards a sustainable development is still not evident. In the near future, the convergence of new technologies will force the replenishment rate and increase the scarcity of resources, especially in energy, food and water, which will affect every facet of the community [13]. Therefore, sustainability is a key approach towards dealing with the changing dynamics of the human element, society and ecology. Design domain must incorporate the sustainability approach into design process of artifacts and systems in order to provide a positive-pull to society, which would create a well-maintained and or ongoing (self-sustained) system that does not degenerate resources of future generations while providing good quality of living to present generation [13].

\section{Human Factors in Product Design}

\subsection{Human Factors}

The main focus of Human Factors is design of optimal products and systems. This involves developing both research and application framework to build a knowledge base about human needs, abilities and limitations, then apply this knowledge to the design of human-machine system products or services. Human Factors domain utilizes the knowledge gained from different disciplines and utilize this knowledge for design and evaluation products, services, tasks, environments and systems which consider human needs, abilities and limitations. Therefore Human Factors is both a theoretical and an applied science and strongly associated with engineering due to its design focus $[10,14]$.

Different range of requirements must be satisfied for a compatible human-artifact systems. Therefore Human Factors design can be defined as mapping from humanartifact systems compatibility (including capabilities and limitations) to system requirements (including ecology, cost, time, safety). Human Factors design practices must consider human capabilities and limitations with the artifact systems while satisfying design constraints such as environmental awareness, cost, time, safety and regulations [10]. This would extend the scope of Human Factors from contemporary 
design to a more hybrid form of a design, which would bring a more holistic approach to design of products and systems $[10,14]$.

\subsection{Importance of Human Factors in Design Domain}

Literature review reveals that most of the designers do not regard Human Factors Engineering (HFE) principals during design of products $[3,10,15]$. Also, not enough fundamental interest is paid to HFE principles comparing to mechanical engineering or software programming. However, if designers employ a better design practice through HFE and follow a Human Centered Design approach, failures due to poor design practice would decrease [16]. Only two percent of decisions during design process were logical, and the rest $98 \%$ were decisions based on past experience and intuition. This case-based trial-and-error procedure and requires modification design slightly and evaluate results to see any progress is made. During this associative and negotiating cognitive work, human needs, abilities and limitations are omitted or not get sufficient attention [10,14]. It is possible to put many human characteristics or outcomes (such as comfort, fatigue) into a systematic and a structured order. This may eliminate some of the irrational nature in conceptual design and product innovation and keep important "measurable" human design elements within attention throughout the product development cycle [10,14].

\section{$4 \quad$ Human Factors to Assist Sustainability and Human Centered Design}

Information provided in above sections demonstrates that there are many commonalities between Sustainability and Human Centered Design and that both can be considered within the Human Factors domain. Each focuses on the well-being of human and attempts to improve product design and user outcomes. Therefore, it is natural for Human factors engineers to encompass ecological issues during design process and a must for consideration of safe, comfortable and sustained interaction with individuals and other elements of the system [17]. Human factors engineers are also prepared to understand, theorize and implement sustainability concepts in the design process [17]. It is suggested that Human Factors might provide a holistic approach to product design and assist in integrating Sustainability concepts with Human Centered Design [12].

\subsection{A Sustainable Human Centered Design Framework Based on Human Factors}

The proposed design framework not only integrates Design, Engineering and Technology, it also provides a more systematic understanding of human element and a related domain, Sustainability, within the product development process. This multidisciplinary approach also allows a modular integration to second and third party domains (including Sustainability and Education) that can provide a knowledgebase 
and methods to support product development. The emphasis in this framework is to introduce Human Factors aspects of product design early in the concept generation phase, provide a modular platform to allow integration of new technologies and to enhance the form, functionality, safety, usability, sustainability and marketing of products. Digital Human Modeling (DHM) is proposed as a middle-ware, which would establish a design medium between these domains. The key contribution of this framework in the context of the Human Factors literature is that DHM is used here as a design medium between related domains rather than solely as an ergonomics evaluation tool. The quantitative nature of DHM and its flexibility in integration with digital design tools and platforms make DHM a unique design tool, which could provide a seamless integration with concurrent engineering platforms and form a linkage between technical and non-technical aspects of concept product development.

\subsection{Digital Human Modeling}

The complex functions of the human body, both physical and cognitive, can be digitally represented, simulated and/or analyzed through DHM tools [18]. DHM uses digital humans as representations of workers inserted into a simulation or virtual environment to facilitate the prediction of performance and/or safety. Also, it includes visualizations of the human with the math and science in the background [19,20]. DHM helps organizations design safer and efficient products while optimizing the productivity and cost [21]. Engineering design practices that utilize DHM have the potential to enable engineers to incorporate human factors engineering principles earlier in the design process [3,22]. Traditionally, DHM applications have been utilized by manufacturing and design industry. One of the first digital human modeling applications was implemented by the U.S. military for cockpit design in which virtual drivers were used to assess the safety and the performance of the prototyped vehicles. This minimized the need of expensive and bulky physical mockups [3,18].

\subsection{A Brief Introduction to Framework}

Above integration provides a platform for integrating Human Factors in early phases of the design process, and acts as a medium to provide a bridge between a wide range of technical and non-technical domains. In the core of the framework DHM is used as both an analytical design/analysis tool as well as a communication medium between contributors in each different stage of the design. The Human Centered product development in this study keeps user needs, abilities and limitations in focus and Human Factors methods and tools encompass different domains related to design process integrated within the framework. The variations and combinations of methods and technologies inside the framework depend on the nature of the product in design. Sustainability, in this case, can be added to the design cycle as a sub-category of the Human Factors domain (Fig.2). Digital Human Modeling makes this integration possible. Ecology-human related components of the design content can be carried into the design cycle by using Digital Human Modeling tools and methods. 
The framework discussed in above is demonstrated with a brief study in vehicle design domain. Figure 3 demonstrates how a concept Formul-1 race car was developed from scratch. The product development started with 2D digitalized sketching and then 3D models were generated. At Stage 2 (Human Factors), DHM tools were utilized to check driver's posture and biomechanics assessment was run to generate comfort study. Meanwhile, materials used in this process (recycled materials or materials coming from energy efficient process/factories) were integrated to the sustainable development process. After defining acceptable cockpit geometry and driver posture, engineering analyses were applied to check structural integrity of the cockpit and chassis as well as control systems. At the very end stage a digital prototype of was rendered (Fig.3).

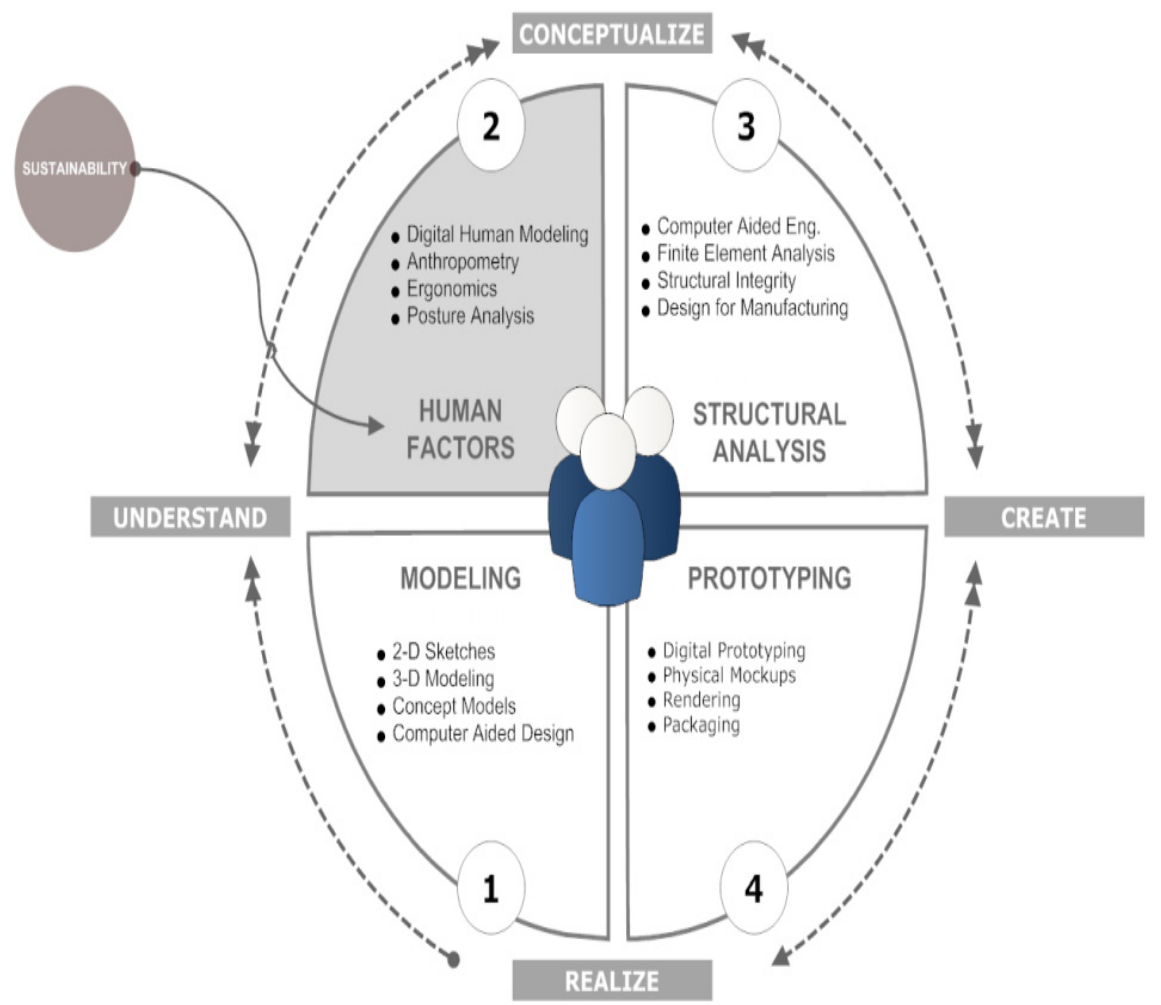

Fig. 2. The Nature of Design (Overall)

In this introductory case example which was considered after actual design, there was no effective means of real integration in terms of Sustainability (green materials) with the actual design process. Parts of this case are included in order to demonstrate the potential of the framework to support Sustainable Human Centered Design process. It is authors' belief that a real holistic integration based on the framework is possible and the use of Human Factors tools and methods would form an ideal platform for future design challenges. 


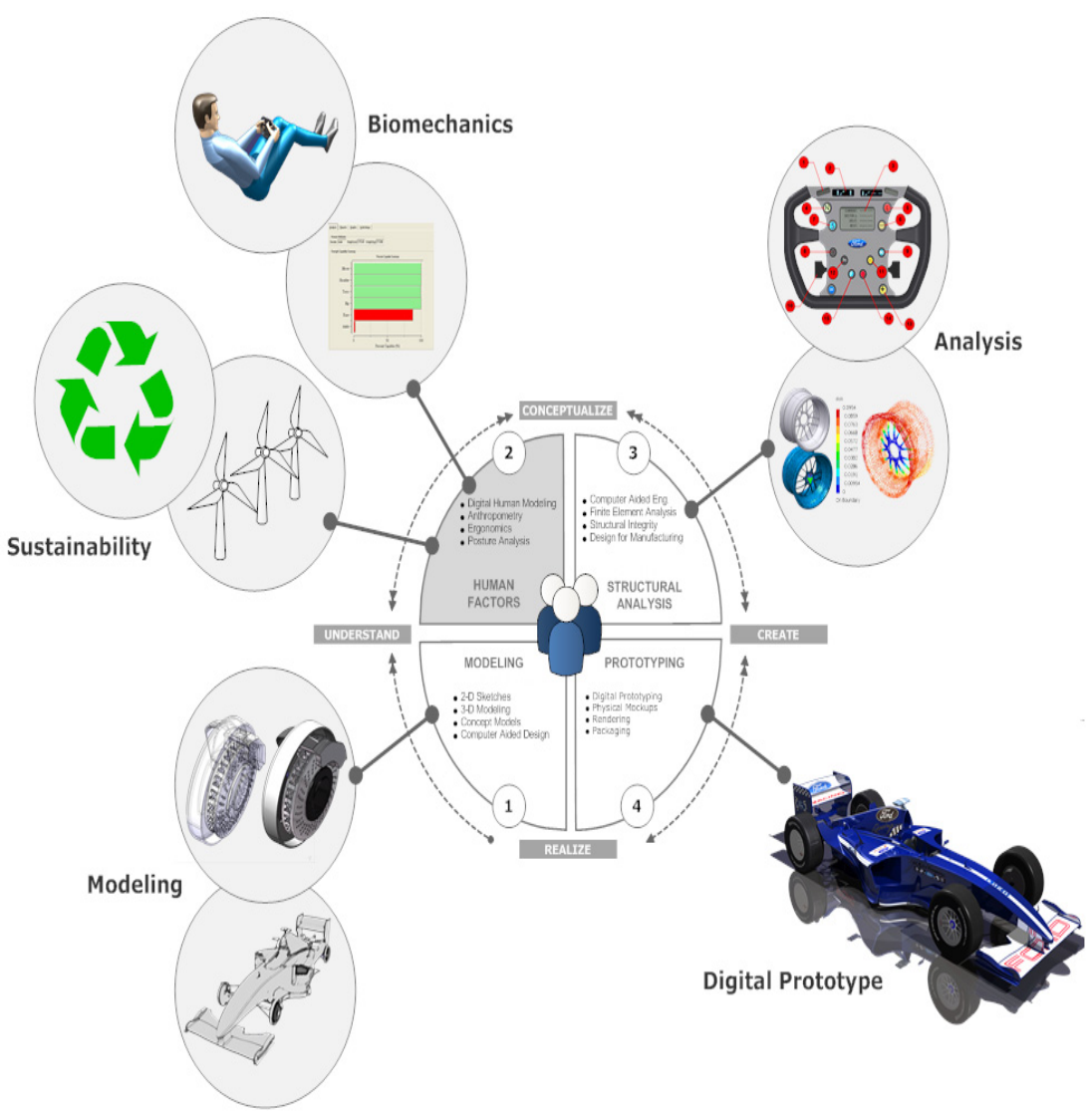

Fig. 3. Formula 1 Concept Design Study by Utilizing the Framework (Sketch-to-Analysis)

\section{References}

1. Raymer, D.P.: A Conceptual Approach. In: Raymer, D.P. (ed.) Design - A Seperate Discipline, pp. 1-10. American Institute of Aeronautics and Astronautics (1989)

2. Holmes, W.P., Azam, M.A., Hills, P.C.: A Preliminary Study of the Relationship Between Industrial Design and Engineering Design. In: Proceedings of the 1st Conference on Designing Interactive Systems: Process, Practices, Methods, \& Techniques, pp. 105-114. ACM, New York (1995)

3. Chaffin, D.B.: Digital Human Modeling for Vehicle and Workplace Design. Society of Automotive Engineers, Warrendale (2001)

4. Thatcher, A.: Green Ergonomics: Definition and Scope, pp. 1-11 (August 2012) (published online), doi:10.1080/00140139.2012.718371

5. Dekker, S.W., Hancock, P.A., Wilkin, P.: Ergonomics and Sustainability: Towards an Embrace of Complexity and Emergence. Ergonomics, 1-8 (September 2012) (published online), doi:10.1080/00140139.2012.718799

6. Tseng, M.M., Du, X.: Design by Customers for Mass Customization Products. The International Academy for Production Engineering 47(1), 103-106 (1998) 
7. Silveira, G., Borenstein, D., Fogliatto, F.S.: Mass Customization: Literature Review and Research Directions. International Journal of Production Economics 1(13), 1-13 (2001)

8. Wickman, C., Soderberg, R.: Increased Concurrency Between Industrial and Engineering Design using CAT Technology Combined with Virtual Reality. Journal of Concurrent Engineering 11(1), 7-15 (2003)

9. Helander, M.G.: The Human Factors Profession. In: Salvendy, G. (ed.) Handbook of Human Factors and Engineering, 2nd edn., pp. 3-16. Wiley (1997)

10. Karwowski, W.: The Discipline of Ergonomics and Human Factors. In: Salvendy, G. (ed.) Handbook of Human Factors and Ergonomics, 3rd edn., pp. 3-31. Wiley (2006)

11. Nemeth, C.P.: Human Factors Methods for Design: Making Systems Human-Centered. CRC Press (2004)

12. Martin, K., Legg, S., Brown, C.: Designing for sustainability: ergonomics - carpe diem. Ergonomics, 37-41 (October 2012), doi:10.1080/00140139.2012.718368.

13. Karwowski, W.: Building Sustainable Human-Centered Systems: A Grand Challenge for the Human Factors and Ergonomics Discipline in the Conceptual Age. In: Corporate Sustainability as a Challenge for Comprehensive Management, pp. 117-128 (2008)

14. Czaja, S.J., Nair, S.N.: Human Factors Engineering and Systems Design. In: Salvendy, G. (ed.) Handbook of Human Factors and Ergonomics, 3rd edn., pp. 32-49. Wiley (2006)

15. Daams, B.J.: Force Exertion in User Product Interaction. In: Karwowski, W., Marras, W.S. (eds.) The Occupational Ergonomics Handbook, pp. 421-435. CRC Press LLC, Boca Raton (2000)

16. Clarkson, J., Ward, J.: Human Factors Engineering and the Design of Medical Devices. In: Carayon, P. (ed.) Handbook of Human Factors and Ergonomics in Health Care and Patient Safety, pp. 367-383. Routledge, Boca Raton (2006)

17. Marano, A., Di, G., Rossi, E.: Strategies and Arguments of Ergonomic Design for Sustainability, Work. A Journal of Prevention, Assessment and Rehabilitation 41, 3869-3873 (2012), doi:10.3233/WOR-2012-0053-3869.

18. Sundin, A., Örtengren, R.: Digital Human Modeling for CAE Applications. In: Salvendy, G. (ed.) Handbook of Human Factors and Ergonomics, pp. 1053-1078. John Wiley \& Sons Inc. (2006)

19. Demirel, H.O., Duffy, V.G.: Application of Digital Human Modeling in Industry. In: Duffy, V.G. (ed.) Digital Human Modeling, HCII 2007. LNCS, vol. 4561, pp. 824-832. Springer, Heidelberg (2007)

20. Demirel, H.O., Duffy, V.G.: Digital Human Modeling for Product Lifecycle Management. In: Duffy, V.G. (ed.) Digital Human Modeling, HCII 2007. LNCS, vol. 4561, pp. 372 381. Springer, Heidelberg (2007)

21. Yang, J., Abdel-Malek, K., Farrell, K.: The IOWA Interactive Digital Human Modeling Virtual Environment. In: Proceedings of IMECE 2004, ASME International Engineering Congress, The 3rd Symposium on Virtual Manufacturing and Application, Anaheim, CA, pp. 1059-1067 (2004)

22. Demirel, H.O.: Sensory Feedback Mechanism for Virtual Build Methodolgy. Thesis: Unpublished Manuscript. Purdue University, West Lafayette (2008)

23. Porter, J.M., Keith, C., Freer, M.T.: Computer-Aided Design and Human Models. In: Karwowski, W., Marras, W.S. (eds.) Occupational Ergonomics: Principles of Work Design, pp. 479-499. CRC Press (2003) 